\title{
Mars Organic Molecule Analyzer (MOMA) Mass Spectrometer for ExoMars 2018 and Beyond
}

\author{
William B. Brinckerhoff ${ }^{1}$, Veronica T. Pinnick ${ }^{2}$, Friso H. W. van Amerom ${ }^{3}$, Ryan M. Danell ${ }^{4}$, Ricardo D. Arevalo, Jr. ${ }^{1}$,
} Martina S. Atanassova ${ }^{2}$, Xiang Li ${ }^{2}$, Paul R. Mahaffy ${ }^{1}$, Robert J. Cotter ${ }^{5}$, Fred Goesmann ${ }^{6}$, Harald Steininger ${ }^{6}$, and the MOMA Team ${ }^{1-9}$

${ }^{1}$ Code 699, NASA Goddard Space Flight Center, 8800 Greenbelt Rd., Greenbelt, MD 20771, ${ }^{2}$ Center for Research and Exploration in Space Science and Technology (CRESST), University of Baltimore, Howard County, Baltimore, MD, ${ }^{3}$ SRI International, Inc., St. Petersberg, FL, ${ }^{4}$ Danell Consulting, Inc., Greenville, NC, ${ }^{5}$ Johns Hopkins University School of Medicine, Baltimore, MD, ${ }^{6}$ Max Planck Institut für Sonnensystemforschung (MPS), Lindau, Germany, ${ }^{6}$ Laboratoire Interuniversitaire des Systèms Atmosphériques (LISA), Univ. Paris-Est, Créteil, France, ${ }^{7}$ Laboratoire Atmosphères, Milieux, Observations Spatiales (LATMOS), Guyancourt, France, ${ }^{8}$ Laboratoire de Génie des Procédés et Matériaux (LGPM), École Centrale Paris, Chatenay-Malabry, France, ${ }^{9}$ Laser Zentrum Hannover e.V. (LZH), Hannover, Germany.

*Corresponding author email: william.b.brinckerhoff@nasa.gov

veronica.t.pinnick@nasa.gov, friso.vanamerom@sri.com, rdanell@danellconsulting.com, ricardo.d.arevalo@nasa.gov, martina.s.atanassova@nasa.gov, xiang.li@nasa.gov, paul.r.mahaffy@nasa.gov, rcotter@jhmi.edu

\begin{abstract}
The 2018 joint ESA-Roscosmos ExoMars rover mission will seek the signs of past or present life in the near-surface environment of Mars. The rover will obtain samples from as deep as two meters beneath the surface and deliver them to an onboard analytical laboratory for detailed examination. The Mars Organic Molecule Analyzer (MOMA) investigation forms a core part of the sample analysis capability of ExoMars. Its top objective is to address the main "life signs" goal of the mission through detailed chemical analysis of the acquired samples. MOMA characterizes organic compounds in the samples with a novel dual ion source ion trap mass spectrometer (ITMS). The ITMS supports both pyrolysis-gas chromatography (pyr-GC) and Mars ambient laser desorption/ionization (LDI) analyses in an extremely compact package. Combined with the unprecedented depth sampling capability of ExoMars, MOMA affords a broad and powerful search for organics over a range of preservational environments, volatility, and molecular weight.
\end{abstract}

\section{TABLE OF Contents}

1. INTRODUCTION .1

2. MS REQUIREMENTS .......................................2

3. MS DESIGN ......................................................2

4. Performance Testing ................................4

5. CONCLUSIONS ............................................5

REFERENCES.......................................................5

BIOGRAPHY ........................................................5

\section{INTRODUCTION}

The Mars Organic Molecule Analyzer (MOMA), a dual-ion source mass spectrometer-based investigation capable of both pyrolysis-gas chromatography/mass spectrometry (pyrGCMS) and laser desorption mass spectrometry (LDMS), is a key instrument on the Pasteur Payload of the 2018 ExoMars rover mission to seek the signs of past or present life on Mars. When combined with the new two-meter drill depth sampling capability of the ExoMars rover, MOMA affords a broad and powerful search for organics over a range of preservation environments, volatility, and molecular weight. This combination may prove critical, as complex organic compounds are highly susceptible to degradation by cosmic radiation, which is effective to at least one meter depth on Mars [1]. In addition to enabling mission science, MOMA critically informs strategies for both sampling and in situ analysis for Mars Sample Return (MSR), which remains a top goal of the international planetary science community [2].

GCMS and LDMS are complementary approaches to detection of organic compounds and some inorganic species in complex mineralogical samples such as encountered on Mars. GCMS is well suited to characterizing mixtures of small-to-moderate molecular weight, volatile and semivolatile species thermally released into the gas phase by pyrolysis. GC separation is especially important to identify patterns in molecular structure, such as the even-odd carbon number distribution of some biomolecules such as fatty acids. Selective synthesis can be an indicator of biochemistry versus abiotic processes, which tend to yield all statistically-probable structures. In these cases mass spectra alone may be ambiguous given the many interfering fragment peaks produced from mixtures of organics with electron ionization (EI) as used on MOMA and most other flight instruments.

LDMS is sensitive to nonvolatile organics as the short $(\sim 1$ ns) laser pulse is able to desorb and ionize many molecular species intact, even to high kilo-Dalton $(\mathrm{kDa})$ molecular weights. Carboxylic acids, peptides, polycyclic aromatic hydrocarbons, and macromolecular carbon, or kerogen-like hydrocarbons, are detectable through LDMS. These may be of great importance to distinguish the origin of any organics, 
given that the majority of cometary and interplanetary dust that has fallen on Mars is in a macromolecular hydrocarbon structure, and may dominate the abundance profile at the parts-per-million levels by weight in the regolith. In addition, LDMS detects some elemental and mineral oxide and sulfide species, illuminating the host environment of any organics detected in the same point-by-point analysis. The addition of LDMS to GCMS data could also help indicate which species passing through the columns may be structurally related to larger and less-volatile species seen with laser desorption.

MOMA is led by the Max Planck Institute for Solar System Exploration (MPS, PI: Dr. Fred Goesmann). Development of the major subsystems of MOMA depicted in Fig. 1 is shared among the major partners. MPS is responsible for the tapping (sealing) station, pyrolysis ovens, and the $266 \mathrm{~nm}$ pulsed laser (and electronics). The 4-column GC including regulated $\mathrm{He}$ gas, injection traps, thermal conductivity detectors, and a multiplexed valve system is provided by a consortium of French laboratories (Co-PI: Dr. Francois Raulin) and is substantially the same team that provided the Sample Analysis at Mars (SAM) six-column GC now operating on Mars in the Mars Science Laboratory (MSL) rover. The linear ion trap MS, the RF power supply, the turbomolecular vacuum pump, and the two electronics boxes that control all of MOMA, are provided to MPS as a subsystem, termed MOMA-MS, by a NASA/GSFC-led U.S. team partnering with the University of Michigan, Space Physics Research Lab, Battel Engineering, and Creare, Inc., all of which have experience collaborating on the development of SAM. The overall MOMA investigation was recently summarized by Steininger et al. [3]. This report focuses on the MS.

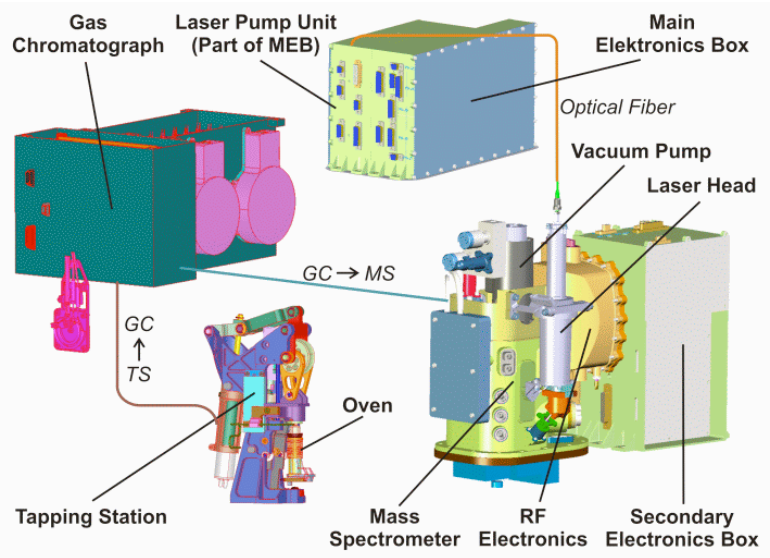

Fig. 1. Overview of MOMA instrument modules. The mass spectrometer and electronics are integrated with a pyrolysis system, including tapping station and ovens, and a fourcolumn gas chromatograph, into the ExoMars rover.

\section{MS REQUIREMENTS}

To achieve both GCMS and LDMS in a common mass spectrometer and remain within the extremely limited mass, power, and volume resources available on ExoMars, a dual- ion source ion trap MS (ITMS) design was adopted. The 70 $\mathrm{eV}$ EI source ionizes compounds entrained in He gas that is split from the pyr-GC effluent and leaked into the MS. The pulsed LDI source produces ions directly from the surface of samples, at Mars ambient pressure, positioned near the MS inlet by the rover's sample processing and delivery system (SPDS) on a refillable tray. This configuration necessitates a tolerance of higher pressures than typical in mass spectrometry, which was partly the basis for selecting an ion trap. The requirements on the ITMS in each of the operating modes are summarized in Table 1. These must be met across a range of Mars surface/rover ambient conditions (i.e., 4 to 8 Torr and $-40^{\circ} \mathrm{C}$ to $+20^{\circ} \mathrm{C}$ ) to ensure the full science return over the 180 sol operational lifetime of the mission. In addition to these figures of merit, the MS must record spectra quickly without long settling or pumping times. For example, individual full $\mathrm{m} / \mathrm{z}$ range scans must be taken at $10 \mathrm{~Hz}$ or faster in pyr-GC mode to capture the shapes of fast $(\sim 1 \mathrm{sec})$ peaks eluting from the columns.

Table 1. Summary of MOMA-MS Requirements

\begin{tabular}{|l|c|c|}
\hline $\begin{array}{l}\text { Performance } \\
\text { Specification }\end{array}$ & pyr-GC mode & LDI mode \\
\hline Mass range & $50-500 \mathrm{Da}$ & $50-1,000 \mathrm{Da}$ \\
\hline $\begin{array}{l}\text { Resolution } \\
\text { (FWHM) }\end{array}$ & $\leq 1 \mathrm{Da}$ & $\begin{array}{c}\leq 2 \mathrm{Da} \text { from } 500 \\
\text { to } 1,000 \mathrm{Da}\end{array}$ \\
\hline Sensitivity & $\begin{array}{c}\leq 1 \mathrm{pmol} \\
(\mathrm{SNR} \geq 10)\end{array}$ & $\begin{array}{c}\leq 1 \mathrm{pmol} \\
(\mathrm{SNR} \geq 3)\end{array}$ \\
\hline Accuracy & \multicolumn{2}{|c|}{$\leq 0.4 \mathrm{Da}$} \\
\hline Drift & \multicolumn{2}{|c|}{$\leq 0.4 \mathrm{Da}$ per experiment } \\
\hline
\end{tabular}

\section{MS DESIGN}

The MS assembly including ion trap, laser, pump, RF supply, and valved LDI inlet assembly is shown in Fig. 2.

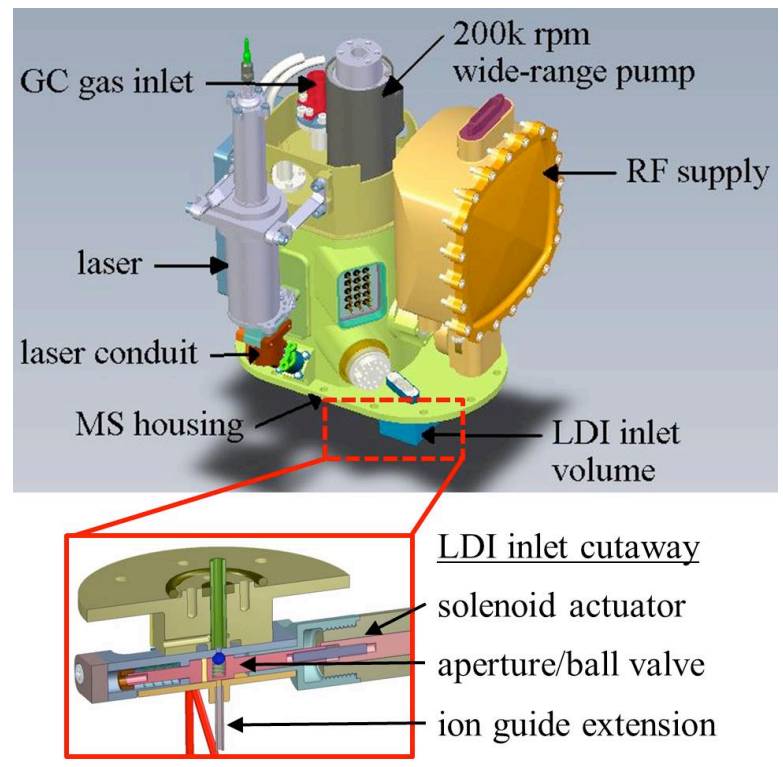

Fig. 2. Current design of the MOMA mass spectrometer. Laser ions from Mars ambient (4-8 Torr) enter the ion trap through the inlet while the valve is open (50-500 msec). 
The assembly shown in Fig. 2 is extremely compact, only about $15 \mathrm{~cm}$ across the base. The MS takes advantage of the double-ended design afforded by a linear ion trap that allows ions to be admitted from either end of the device. The core linear ion trap, depicted in Fig. 3, includes four parallel hyperbolic rods (as in a quadrupole mass filter) and two end-plate electrodes. This configuration is similar to that originally developed by Thermo Scientific for their LTQ and LXQ systems.

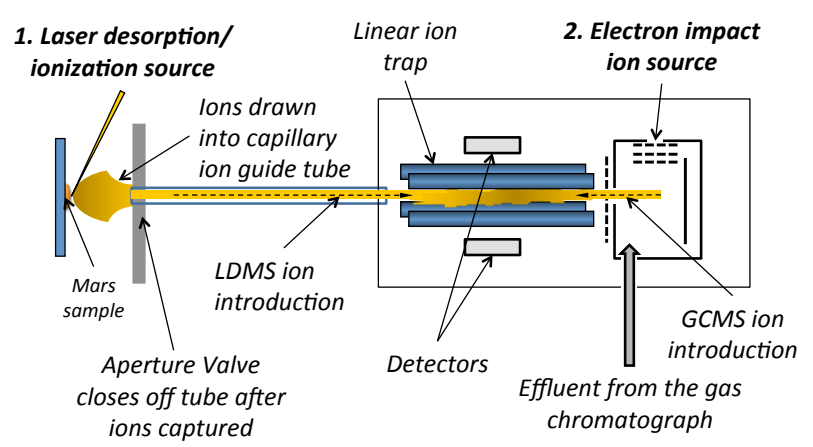

Fig. 3. The double-ended, quadrupole-like geometry of linear ion trap employed in MOMA-MS enables ions from pyr/GC-EI and LDI sources to be analyzed in one volume.

The ion trap operates with a combination of RF and DC potentials applied to the four rods forming an ion trapping volume - hence the device's name. Ions formed in either mode are injected through center holes in the end plates and are trapped in a potential well created by the applied voltages. Trapped ions oscillate in the RF field and exhibit a resonance frequency dependent on their $m / z$ value and several operating parameters of the instrument. To detect the ions and generate a mass spectrum they must be ejected from the trap. A dipolar AC signal is applied to a pair of opposing rods (each containing a narrow longitudinal slit) and the amplitude of the RF voltage is ramped. This causes ions of successively higher mass to resonate with the $\mathrm{AC}$ signal and be ejected through the slits. After ejection the ions are detected with a dynode and channel electron multiplier (CEM). The trap has two detectors (one for each slitted rod) that can be operated individually as redundant single detectors or in parallel for enhanced sensitivity. Mechanical precision requirements for the linear trap are comparable to quadrupole mass filters with which our team has much experience. The Goddard-built miniature ITMS (Figs. 4, 5) is a factor of 3-4 times smaller than the commercial state-of-the art analyzer, allowing lower voltages to be used to achieve the same $\mathrm{m} / \mathrm{z}$ range.

\section{Electron Ionization (GCMS mode)}

The EI source used in MOMA-MS (Fig. 5) is very similar to EI sources used with the quadrupole mass spectrometers (QMS) in SAM and the Mars Atmosphere and Volatile Evolution (MAVEN) Neutral Gas and Ion Mass Spectrometer (NGIMS). The source contains two redundant $\mathrm{W}$-Re filaments producing $70 \mathrm{eV}$ electron beams with up to $50 \mu \mathrm{A}$ of electron current that ionize neutral pyr/GC effluent delivered directly into the closed ionization volume. The electron and ion beams are gated such that ions are only generated and injected into the instrument when desired, which eliminates noise and stray ions on the detector. The LIT is operated with a background pressure of $\sim 0.5 \mathrm{mTorr}$ in GCMS mode which provides optimum instrument sensitivity and mass spectral performance.

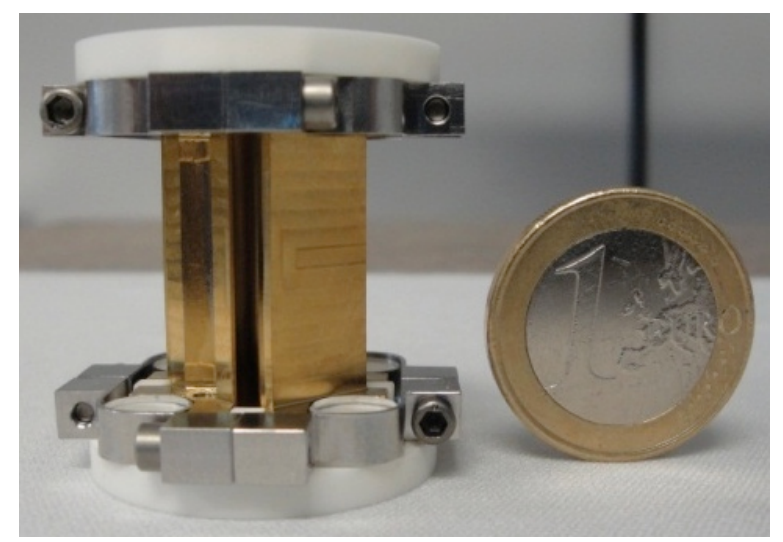

Fig. 4. The MOMA-MS miniaturized LIT mass analyzer.

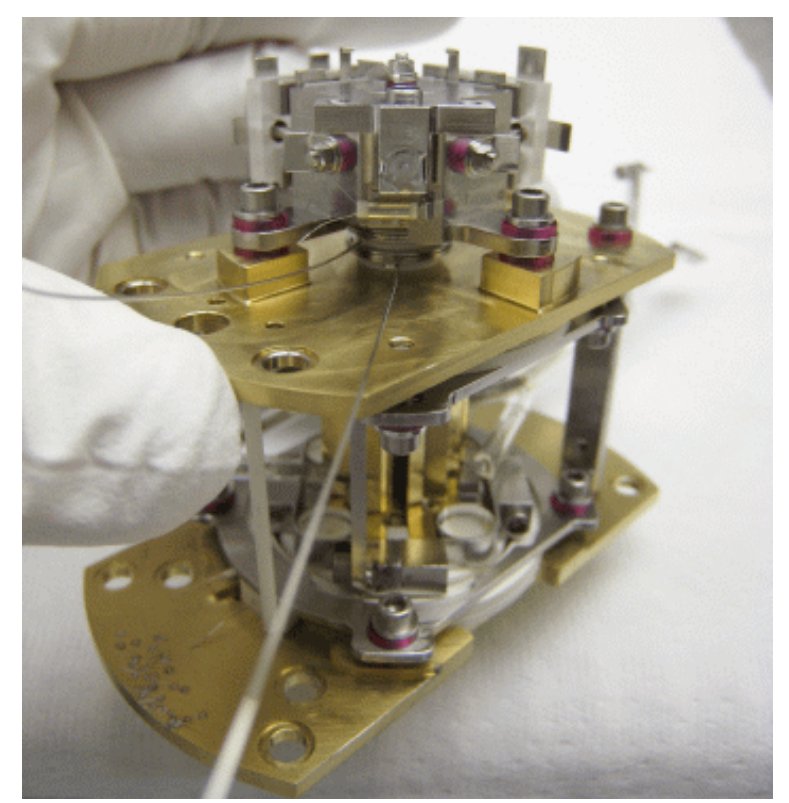

Fig. 5. MOMA ITMS with linear trap between the plates and SAM-heritage EI source above the upper plate.

\section{Laser Desorption/Ionization (LDMS mode)}

The LDI ion source used in MOMA-MS was largely a new development, although it is based on the discontinuous atmospheric pressure ionization (DAPI) scheme of Gao et al. [4]. The source uses a custom, fast-acting, aperture valve and coupled ion transfer tube (Fig. 2). In its application on MOMA-MS this configuration has been built at flight scale and has survived life testing to over $10^{5}$ cycles.

To perform LDI a frequency-quadrupled (266 nm) passively Q-switched Nd:YAG laser is focused at 45 degrees incidence onto the sample surface held at Mars ambient 
pressure (4-8 Torr, primarily $\mathrm{CO}_{2}$ ), which is positioned at a point 3-5 $\mathrm{mm}$ below the entrance of the ion transfer tube (Fig. 2). The aperture valve, ]mounted in the middle of the tube, is opened for a few hundred msec causing the ion trap pressure to rise quickly to several tens of mTorr. With the valve open, the laser fires a burst of pulses desorbing and ionizing atomic and molecular species directly from the surface. A potential applied to the inlet draws ions across the gap toward the tube. Near the tube entrance, ions are drawn in via gas flow entrainment carrying them to the ion trap (a distance of $3 \mathrm{~cm}$ ). Following the last laser pulse, the valve is closed and the ion trap chamber pumps down via the vacuum pump to 0.1-0.5 mTorr in several seconds (the precise time depends on a number of experimental factors). The ions are readily trapped for tens of seconds at high pressures, a strong benefit of using an ion trap for this application. The detector high voltages are then switched on and the ions are ejected and detected.

Laser pulses of up to $\sim 200 \mu \mathrm{J}$ focused to a spot size of 0.5 $\mathrm{mm}$ diameter or less yield intensities of tens to hundreds of MW $\mathrm{cm}^{-2}$ which cover a wide range of desorption thresholds at $266 \mathrm{~nm}$ for mineral substrates even into the translucent silicate domain. Detectable fractions of the organic moieties desorbed from these substrates can survive intact along with their multi-photon ionization fragments, permitting their characterization even at sub-pmol $\mathrm{mm}^{-2}$ concentrations in some cases. Operating the LDI mode over a range of laser energies, afforded by temperature tuning of the passively Qswitched laser crystal, can reveal correlations between fragments and parent molecules, supporting organic identifications in analysis of complex mixtures.

\section{Turbomolecular Pump}

The baseline vacuum pump for MOMA-MS is the new 200 krpm hybrid turbomolecular drag pump from Creare, Inc. that is a further miniaturized model of their $100 \mathrm{krpm}$ Wide Range Pump (WRP) now operating successfully on Mars in the SAM suite. These 4-5 liter/sec pumps exhaust directly to the Mars ambient atmosphere, obviating the need for a backing stage pump. The operating requirements on the MOMA pump are quite different than those of the SAM WRP, given that the MOMA pump reduces pressure from Mars ambient down only to 0.5 mTorr (rather than below 1 $\mu$ Torr for the SAM QMS), and in LDI mode is cycled to over $10 \mathrm{mTorr}$ with every valve opening to produce one mass scan. However we have found that the same basic design of pump is able to withstand the MOMA requirements and still meet the science objectives for sensitivity, which require the ITMS to be evacuated within $\sim 4$ seconds following closure of the aperture valve to retain and detect as little as $1 \mathrm{pmol} \mathrm{mm}$ of analyte. The MOMA pump is currently in a bearing optimization and lifetime test program that will conclude in early 2013.

\section{Performance Testing}

The MOMA mass spectrometer flight-like breadboard has been tested against performance specifications and optimized within resource and schedule constraints of the ExoMars mission. All of the requirements on the ITMS (Table 1) have been met. The mass resolution and limit of detection specifications have been established through simplified testing of reference compounds, which provide reproducible sampling conditions for the ITMS (Fig. 6).

The mass range has been checked with known highmolecular weight species such as the peptide Angiotensin II $(\mathrm{m} / \mathrm{z}$ 1047) and with cluster-forming sources such as $\mathrm{NaI}$ (Fig. 7), which demonstrates uniform sensitivity to organics exceeding the goal of $2000 \mathrm{Da}$. High molecular weight peaks in complex samples such as Mars analogs are now being systematically studied in a round robin campaign using our commercial (Thermo) linear ion trap and other instrumentation. Initial assessment of MOMA-like protocols applied to organic-bearing Antarctic sediments was presented in Bishop et al., 2012 [5].
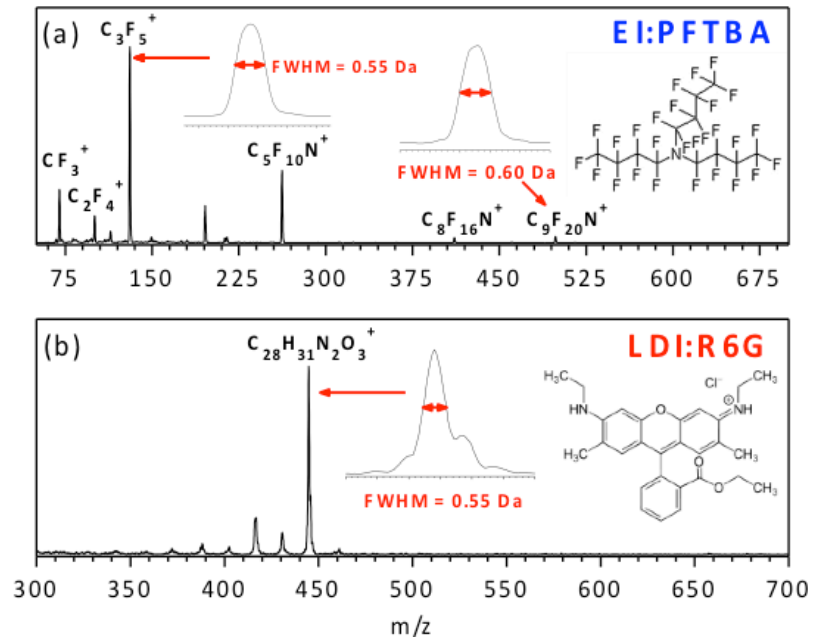

Fig. 6. MOMA-MS meets specifications for mass resolution and detection limits in EI (a) and LDI (b) modes. PFTBA: perfluorotributylamine $\left(\mathrm{CF}_{3}\left(\mathrm{CF}_{2}\right)_{3}\right)_{3} \mathrm{~N}$; R6G: Rhodamine $6 \mathrm{G}$ $\mathrm{C}_{28} \mathrm{H}_{31} \mathrm{~N}_{2} \mathrm{O}_{3} \mathrm{Cl}, 1$ pmol loaded on sample plate.

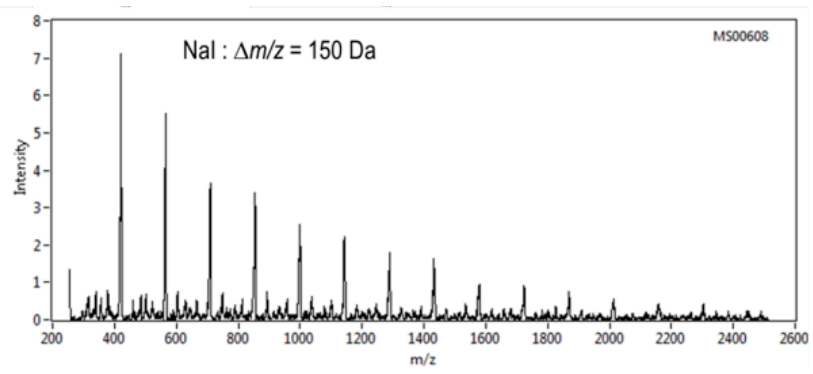

Fig. 7. Mass range of cluster-producing NaI in LDMS mode. Spectrum compares favorably with data from a highperformance commercial (Thermo LTQ) ion trap analysis.

Structural analysis of ions via tandem mass spectrometry (MS/MS) is a useful and somewhat unique benefit of using an ion trap mass spectrometer for planetary missions. A typical MS/MS experiment proceeds as follows. First, the ion trap is filled with ions from LDI. The ions are trapped 
and all but a single mass of interest is ejected from the trap using a notch in either the ejection frequency or voltage where the ion of interest is stable. The ion is then excited and fragmented to form product ions. These ions are retrapped and scanned out and detected as in a normal mass scan. The spectra in Fig. 8 validate each step in the sequence for the peptide Angiotensin II. Here we demonstrate the trapping and isolation of the parent molecular ion $\mathrm{m} / \mathrm{z} 1047$, then its subsequent excitation and fragmentation. This also represents the sensitivity requirement for $\mathrm{MS} / \mathrm{MS}$ mode, which is $10 \mathrm{pmol} \mathrm{m^{-2 }}$ Angiotensin II with SNR $>10$.
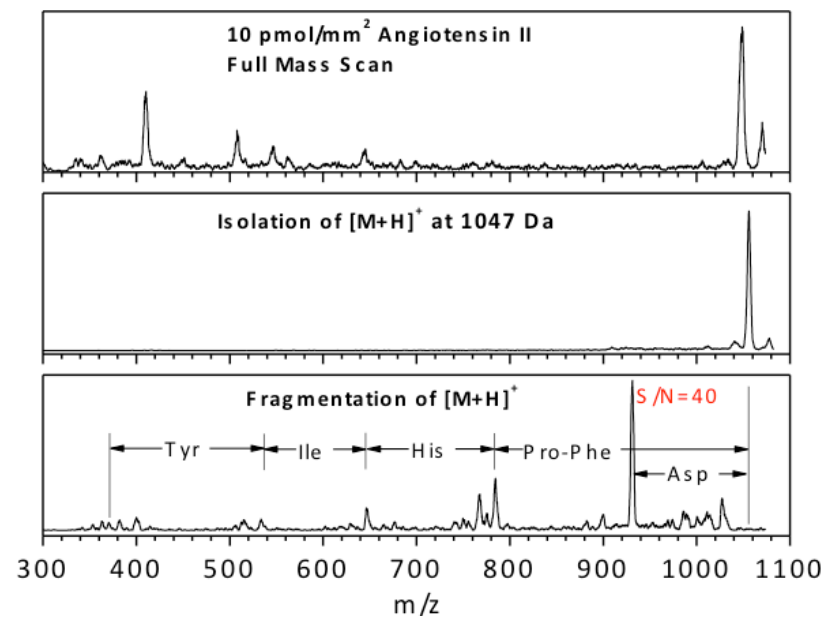

Fig. 8. MS/MS LDI limit of detection demonstration at 10 pmol/ $\mathrm{mm}^{2}$ of peptide Angiotensin II in di-hydroxybenzoic acid (DHB) matrix on MOMA-MS.

\section{Conclusions}

MOMA-MS has met or exceeded its design specifications and has passed its subsystem preliminary design review (PDR) in Dec. 2012. A MOMA-level PDR is planned for spring, 2013 with the ExoMars rover payload PDR to follow shortly thereafter. Brassboard Main Electronics Box and the Secondary Electronics Box electronics have been developed and are undergoing testing with the breadboards. An engineering test unit (ETU) of the mass spectrometer, with fully flight-like housing, RF, and laser configuration will be developed and tested in 2013. The ETU will also be integrated with a flight-like GC prototype for full-up GCMS campaigns in 2013 to validate the preliminary design and prepare for a critical design review (CDR) in early 2014. The MOMA-MS subsystem flight model is planned to deliver to MPS in Dec. 2015, well in advance of the 2018 launch owing to the multiple nested levels of integration and testing of this highly-specialized mission payload.

ExoMars presents a unique opportunity to examine surface and subsurface materials on Mars with a new and unique tool, the MOMA-MS dual source linear ion trap instrument under development with NASA support. As of this writing, the MOMA-MS team has been given the go-ahead by
NASA Headquarters to continue support of the ExoMars mission with this investigation as part of the overall Mars Exploration Program. The MOMA-MS development in general represents a substantial capability that is ready to be brought to flight opportunities in support of the next round of Mars exploration, in the post-MSL era where very careful organic analyses will be needed with an eye to selecting those samples of highest value for eventual return to Earth. The highly-miniaturized MOMA-MS instrumentation would thus be an asset in the MSR campaign.

\section{REFERENCES}

[1] Pavlov, A. A., G. Vasilyev, V. M. Ostryakov, A. K. Pavlov, and P. Mahaffy (2012), Degradation of the organic molecules in the shallow subsurface of Mars due to irradiation by cosmic rays, Geophys. Res. Lett., 39(13), L13202, doi:10.1029/2012GL052166.

[2] National Research Council, Space Studies Board, Committee on the Planetary Science Decadal Survey (2011), Vision and Voyages for Planetary Science in the Decade 2013-2022, The National Academies Press, Washington, D.C.

[3] Steininger, H., E. Steinmetz, W. B. Brinckerhoff, P. R. Mahaffy, R. J. Cotter, F. Raulin, F. Goesmann, C. Szopa, and the MOMA team (2012) Mars Organic Molecule Analyzer (MOMA) onboard ExoMars 2018, Proc. International Workshop on Instrumentation for Planetary Missions (IPM-2012), Oct. 10-12, Oral Presentation.

[4] Gao, L., R. G. Cooks, and Z. Ouyang (2008), Breaking the pumping speed barrier in mass spectrometry: Discontinuous atmospheric pressure interface, Analytical Chemistry, 80(11), 4026-4032, doi:10.1021/ac800014v.

[5] Bishop, J. L., H. B. Franz, W. Goetz, D. F. Blake, C. Freissinet, H. Steininger, F. Goesmann, W. B. Brinckerhoff, S. Getty, V. T. Pinnick, P. R. Mahaffy, and M. D. Dyar (2012), Coordinated analyses of Antarctic sediments as Mars analog materials using reflectance spectroscopy and current flight-like instruments for CheMin, SAM and MOMA, Icarus, doi:10.1016/j.icarus.2012.05.014.

Biographies

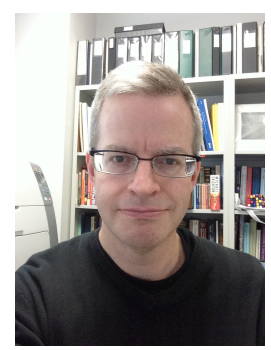

William Brinckerhoff is a senior space research associate at NASA's Goddard Space Flight Center. He received his Ph.D. in Physics from the Ohio State University. His current research interests include development of novel miniature mass spectrometers and sample handling systems for planetary missions, synthesis of organic compounds in the interstellar 
medium, and the habitability of Mars. He serves as a Co-I on MSL/SAM and project scientist for the MOMA ion trap MS on ExoMars.

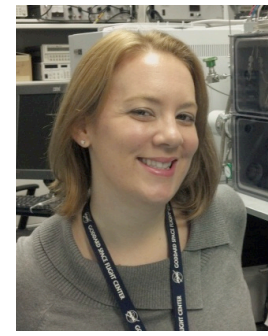

Veronica Pinnick is a Research Associate at NASA Goddard Space Flight Center through the Center for Research in Space Science and Technology at the University of Maryland, Baltimore County. She received her B.A. in Chemistry from Minot State University (2004) and her Ph.D. in Analytical Chemistry from Texas A\&M University (2009). She held a two year post-doctoral fellowship in instrumentation development at Johns Hopkins University School of Medicine. Upon joining the Planetary Environments Lab at NASA GSFC, her current research interests include development of instrumentation and methodology to sample non-volatile organics in ambient planetary environments.

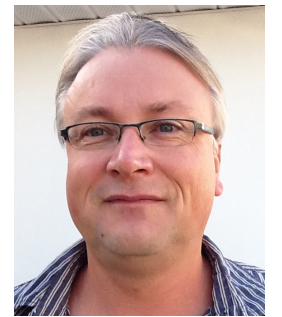

Friso H. W. van Amerom is a senior Researcher at SRI International. He received his B.S. in organic chemistry at chemistry college, Utrecht (1995). He finished a M.S. in physical chemistry (1998) at the VU in Amsterdam at the laser centre (Prof. S. Stolte). Then he studied at University of Amsterdam (Institute of Mass Spectrometry) as Ph.D. student under Prof. N.M.M. Nibbering (1998). He became a Research Associate at University of South Florida (2002) where he specialized in mass spectrometer development. He moved on to work for SRI International (2008). His interests are miniaturization and development of new mass spectrometer technologies, MEMS fabrication and mass spectrometer electronics.

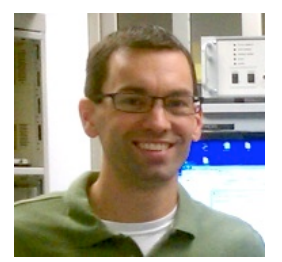

Ryan M. Danell is the President and founder of Danell Consulting, Inc. and specializes in mass spectrometer instrument simulation, design, and control. His primary experience is with trapping and quadrupole based instruments. His research interests are focused on enabling high performance instrument operation through unique designs resulting in novel solutions to current and future analytical problems. Dr. Danell received his Ph.D. in Analytical Chemistry in 2001 from the University of North Carolina at Chapel Hill.

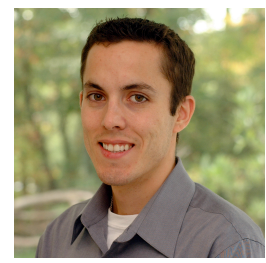

Ricardo Arevalo Jr. is a Research Space Scientist at NASA Goddard Space Flight Center with expertise in laser ablation (LA) and laser desorption/ionization (LDI) mass spectrometry. His current research interests are focused on understanding planetary differentiation and the chemical/thermal evolution of planetary bodies through a variety of geochemical proxies, including trace elemental and isotopic compositions of terrestrial and meteoritic basalts and mantle-derived materials. He received his Ph.D. in Geology in 2010 from the University of Maryland, where he currently serves as an Adjunct Assistant Professor.

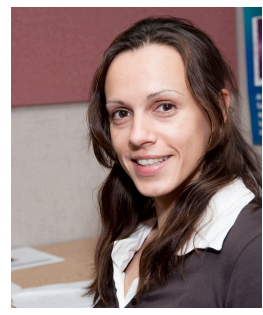

Martina Atanassova is an optical engineer at NASA Goddard Space Flight Center, involved in instrument development and optical analysis for variety of scientific space instrumentation. Her current efforts involve the development of laser desorption mass spectrometry instruments utilizing high power UV lasers and beam steering opto-mechanical design. She received her Ph.D. in Optics from College of Optics and Photonics (CREOL) at University of Central Florida in 2005. She held various postdoctoral and industrial positions around Europe and USA prior to joining NASA Goddard Space Flight center in 2008.

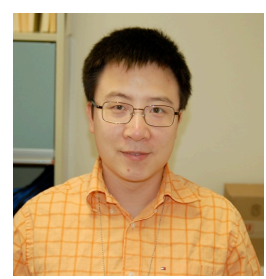

Xiang $\boldsymbol{L} i$ received his B.S. in Chemistry from the Peking University, China in 2003, and Ph.D. in Physical Chemistry from the Johns Hopkins University in 2009. He has been a Research Associate with a joint appointment at the University of Maryland, Baltimore County and NASA Goddard Space Flight Center since 2011. His research focuses on the detection of trace element and astrobiologically relevant organic molecules in planetary systems, like Mars. He is especially interested in the development of Time-of-Flight and Ion Trap mass spectrometers with various ionization and ion gating techniques.

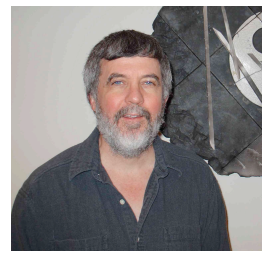

Paul Mahaffy is a planetary scientist at NASA Goddard Space Flight Center. His interests are in chemical and isotopic composition of planetary atmospheres and surfaces and how these inform us regarding the history of these environments. He has participated in several planetary missions including the Galileo Probe measurements of the atmospheric composition with a mass spectrometer. He is presently the Principal Investigator on the SAM suite investigation on the Curiosity rover that is exploring the atmosphere and volatiles in solid samples in Gale Crater on Mars. Paul Mahaffy is also the lead on orbital mass spectrometers to be delivered to lunar and Mars orbit. 


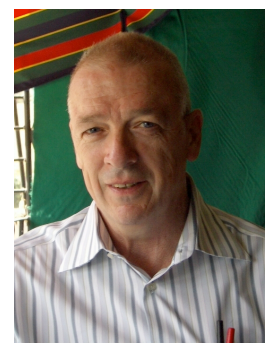

Robert J. Cotter is Professor of Pharmacology and Molecular Sciences at the Johns Hopkins University School of Medicine and Director of the Middle Atlantic Mass Spectrometry Laboratory. He has designed a number of miniaturized and portable time-of-flight mass spectrometers and ion traps, and has been involved in the earliest designs of the MOMA instrument. His expertise also includes laser desorption and applications of mass spectrometry to the analysis of biological samples.

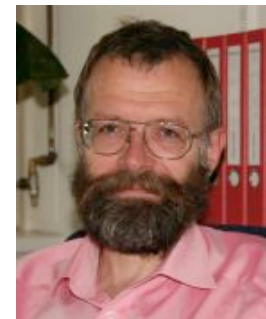

Fred Goesmann is a senior scientist at the Max-Planck-Institut für Sonnensystemforschung (MPS) in Lindau, Germany. He serves as PI of the MOMA investigation on the ExoMars rover as well as PI of the COmetary SAmpling and Composition (COSAC) experiment on the Rosetta mission to Comet 67P/ChuryumovGerasimenko.

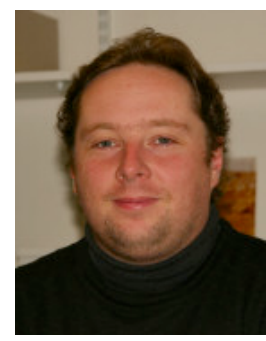

Harald Steininger is a scientist at the Max-Planck-Institut für Sonnensystemforschung (MPS) in Lindau, Germany. He received his Ph.D. at the Ludwig-Maximilians-Universität Munich for work on catalysed aminolysis in 2006. His current research interests include the organic environment on Mars. He is the project scientist and the planetary protection representative of the MOMA investigation on the ExoMars rover. He is responsible for the development of measurement protocols for the MOMA instrument especially for the pyrolysis and derivatization modes. 
